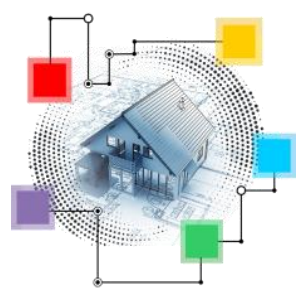

\title{
GERENCIAMENTO DAS PRESSÕES E CONTROLE DAS PERDAS REAIS NOS SISTEMAS DE DISTRIBUIÇÃO DE ÁGUA: UM MAPEAMENTO SISTÉMICO
}

\section{THE MANAGEMENT OF PRESSURES AND CONTROL OF REAL LOSS IN WATER DISTRIBUTION SYSTEMS: A SYSTEMIC MAPPING}

\author{
MORAIS, Lucas S.R. de ${ }^{1}$; PAULA, Heber M. ${ }^{2}$ \\ ${ }^{1}$ Universidade Federal de Goiás, Regional Catalão, Mestrando do PPG em Engenharia Civil, Av. \\ Dr. Lamartine Pinto de Avelar, 1120, Setor Universitário, Catalão, Goiás, \\ lucas.salomao@ufg.br \\ ${ }^{2}$ Universidade Federal de Goiás, Regional Catalão, Prof. do PPG de Modelagem e Otimização e \\ PPG em Engenharia Civil, heberdepaula@ufg.br
}

\begin{abstract}
RESUMO
Os índices de perdas reais nos sistemas de distribuição de água no mundo e no Brasil ainda são bem altos. Nos últimos anos em que as mudanças climáticas provocaram grandes secas e períodos de racionamento e escassez de água nas grandes cidades, o tema ganhou destaque. Esses vazamentos geram desperdício e trazem grandes prejuízos ambientais, econômicos e sociais. O objeto de estudo deste artigo é a revisão de literatura do gerenciamento de vazamentos e controle das pressões nos sistemas de distribuição de água, a fim de catalogar, por meio das bases de dados indexadas, as contribuições científicas relacionadas ao tema, a partir de palavras-chave pré-determinadas. A utilização do Mapping Study proporcionou a catalogação de todo o material, a classificação por diversos aspectos e a análise das tendências de estudo e as lacunas existentes, que são oportunidades de novas pesquisas. Pelos resultados obtidos, observou-se que são oriundos da Europa a maior parte dos artigos relacionados ao tema. $\mathrm{O}$ uso das válvulas redutoras de pressão é o método mais utilizado para se controlar os vazamentos e a pressão dos sistemas. Os problemas de otimização relacionados aos modelos de calibração de rede e os aspectos e impactos ambientais quando da implantação do controle das perdas reais são as vertentes de pesquisa menos exploradas.
\end{abstract}

Palavras-chave: Pressão, Perdas Reais, Vazamento, Sistemas de Distribuição de Água.

\begin{abstract}
The indices of real losses in water distribution systems in the world and in Brazil are still very high. In recent years, when climate change has caused great droughts and periods of rationing and water scarcity in large cities, the theme has gained prominence. These leaks generate waste and bring great environmental, economic and social damages. The purpose of this article is to review the literature on leakage management and pressure control in water distribution systems, in order to catalog, through indexed databases, the scientific contributions related to the theme, from words keys. The use of the Mapping Study provided the cataloging of all the material, the classification by several aspects and the analysis of the study trends and the existing gaps, which are opportunities for new research. From the results obtained, it was observed that most of the articles related to the topic came from Europe. The use of pressure reducing valves is the most widely used method to control leaks and pressure systems. The optimization problems related to the network calibration models and the environmental aspects and impacts when implementing real loss control are the least explored research areas
\end{abstract}

Keywords: Pressure, Real Losses, Leakage, Water Distribution Systems. 


\section{INTRODUÇÃO}

As perdas de água nos sistemas de distribuição podem ser dividias em três tipos: as perdas aparentes (falhas de cadastro ou erros de macro ou micromedição), consumos não autorizados (desvios e fraudes) e as perdas reais (vazamentos visíveis, invisíveis ou inerentes) (ABES 2013). Dentre esses tipos de perdas, as perdas reais representam a maior parte, cerca de $70 \%$ (WHO 2001).

O índice de perdas nos sistemas de distribuição de água no Brasil, considerando os dados mais recentes do Sistema Nacional de Informações Sobre Saneamento (SNIS 2016), é de $38,05 \%$. Considerando os diversos avanços tecnológicos já alcançados nas mais diversas áreas de conhecimento, esse índice pode ser considerado bastante elevado.

O excesso da pressão nas redes é uma das principais causas dos rompimentos e da geração dos vazamentos nas tubulações. O uso de Válvulas Redutoras de Pressão (VRP) é uma metodologia bastante utilizada e se mostra viável para a realização do gerenciamento da pressão e das perdas reais nos sistemas (SAMIR et.al (2017).

A utilização de VRP nesse controle é uma opção com ótimo custo-benefício e não exige difíceis técnicas para operação (NICOLINI e ZOVATTO 2009; DARVINI e SOLDINI 2015; SAMIR et.al 2017). A otimização do número dessas válvulas e sua localização são objeto de estudo de vários autores, que fazem uso de técnicas de otimização clássica, métodos bioinspirados (NICOLINI e ZOVATTO 2009; ARAUJO et al. 2006) ou também utilizando Programas Não-Lineares Inteiros Mistos (PECCI, 2015).

\section{OBJETIVOS}

Considerando o cenário mundial do controle de perdas nos sistemas de saneamento e os diversos estudos científicos já realizados, este artigo tem como objetivo principal investigar e expor o cenário das pesquisas relacionadas diretamente com o gerenciamento das pressões e controle das perdas reais nos sistemas de distribuição de água, através do Mapping Study, além de apresentar as perspectivas e possíveis lacunas relacionadas ao tema.

\section{MATERIAIS E METÓDOS}

O Mapping Study é uma metodologia de pesquisa que tem como objetivo mapear os estudos já realizados e que estão em andamento em um determinado assunto, adotando como limitador palavras-chave. Envolve desde o levantamento de material científico em banco de dados, até o tratamento e avaliação, com a classificação das informações e catalogação. Com esses passos, é possível identificar as principais vertentes estudadas, as lacunas existentes, os principais pesquisadores do assunto e onde mais frequentemente esses artigos estão publicados (BAILEY et al. 2007).

A metodologia utilizada para o levantamento da literatura em conformidade com o tema foi o Mapping Study, ou mapeamento sistêmico. Diferentes autores, em suas publicações, 
descrevem como a metodologia consegue identificar os principais trabalhos e caracterizar com qualidade o estado da arte sobre o assunto, como exemplo, pode-se destacar Paula et al., (2016), Rodrigues et al., (2017), Bonfim et al., (2017) e Fernandes et al., (2017).

Na realização do levantamento, as bases de dados bibliográficos selecionadas foram o Portal de Periódicos oferecido pela Coordenação de Aperfeiçoamento de Pessoal de Nível Superior do Ministério da Educação (CAPES, 2018), Compendex (ENGINEERING VILLAGE, 2018) e pelo Scopus (2018). Todos os acessos foram realizados pela rede da Universidade Federal de Goiás.

Considerando a temática a ser revisada e os recursos oferecidos pelas ferramentas de busca, como operadores booleanos e aspas, foram estabelecidas as seguintes palavraschave para realizar a pesquisa: (a) Pressure AND "Water distribution systems" (b) "Real losses" AND "water distribution systems" (c) Leakage AND "Water distribution systems". Em todas as bases aplicou-se o filtro "Periódico revisado por pares" ou "journal article". Devido ao grande número de resultados encontrados, no banco de dados da CAPES foram buscados artigos dos últimos 35 anos. Na pesquisa, foram utilizados filtros de busca para otimizar os resultados. As pesquisas foram realizadas entre os meses de setembro e outubro de 2018.

Após a realização das buscas, foi realizada a leitura dos títulos de cada artigo. Os artigos com títulos aderentes ao objeto da pesquisa foram salvos em listas dentro das próprias páginas das bases. Essas listas foram importadas em formato BibTex para acesso fora da rede de internet. Esses arquivos foram abertos dentro do Programa JabRef®. A partir desse programa, realizou-se a remoção de títulos duplicados e a leitura dos resumos dos artigos selecionados.

Após a leitura dos resumos, foi realizada uma segunda seleção, descartando novamente artigos que não tivessem relação direta com o tema em estudo. Nos que tinham ligação ao assunto, realizou-se a identificação das vertentes e tendências de pesquisa. Por fim, os artigos selecionados foram exportados do programa JabRef® para uma planilha no Microsoft Excel® para tratamento dos dados a partir de filtros, gráficos e tabelas.

\section{RESULTADOS E DISCUSSÕES}

Efetuadas a análise e avaliação dos dados obtidos, estes foram organizados de forma a alcançar os objetivos do Mapping Study. Todos os dados foram organizados em função das palavras-chave pré-determinadas. A Tabela 1 apresenta o número de artigos obtidos dentro das bases de dados científicos na pesquisa inicial, após a análise de título dos trabalhos, depois da remoção das duplicatas e posterior a leitura dos resumos. Ao final, o resultado obtido foi um banco de dados bibliográficos composto de 150 artigos aderentes diretamente à temática do trabalho, divididos entre as três palavras-chave escolhidas. 
TABELA 1 - Resultados obtidos no Mapping Study

\begin{tabular}{|c|c|c|c|c|c|c|}
\hline \multirow{4}{*}{ Bases de Dados } & \multicolumn{6}{|c|}{ Palavras-Chave } \\
\hline & \multicolumn{2}{|c|}{$\begin{array}{l}\text { Pressure AND } \\
\text { "Water distribution } \\
\text { systems" (a) }\end{array}$} & \multicolumn{2}{|c|}{$\begin{array}{l}\text { "Real losses" AND } \\
\text { "water distribution } \\
\text { systems" (b) }\end{array}$} & \multicolumn{2}{|c|}{$\begin{array}{l}\text { Leakage AND } \\
\text { "Water distribution } \\
\text { systems" (c) }\end{array}$} \\
\hline & \multicolumn{6}{|c|}{ Número de Artigos } \\
\hline & Inicial & $\begin{array}{c}\text { Título } \\
\text { Aderente }\end{array}$ & Inicial & $\begin{array}{c}\text { Título } \\
\text { Aderente }\end{array}$ & Inicial & $\begin{array}{c}\text { Título } \\
\text { Aderente } \\
\end{array}$ \\
\hline Periódicos Capes & 403 & 44 & 64 & 14 & 907 & 45 \\
\hline Scopus & 253 & 20 & 25 & 9 & 113 & 20 \\
\hline Compendex & 163 & 18 & 16 & 9 & 405 & 63 \\
\hline Sub-Total & 819 & 82 & 105 & 32 & 1425 & 128 \\
\hline $\begin{array}{l}\text { Artigos selecionados após retirar } \\
\text { repetições }\end{array}$ & \multicolumn{2}{|c|}{67} & \multicolumn{2}{|c|}{48} & \multicolumn{2}{|c|}{120} \\
\hline $\begin{array}{l}\text { Artigos selecionados após leitura } \\
\text { do resumo }\end{array}$ & \multicolumn{2}{|c|}{47} & & 25 & \multicolumn{2}{|c|}{78} \\
\hline Total & \multicolumn{6}{|c|}{150} \\
\hline
\end{tabular}

Fonte: Autoria Própria.

Observa-se que as palavras-chave (b) alcançaram um índice relativo entre os artigos selecionados após a análise final e os escolhidos no levantamento inicial de 23,81\%, enquanto que as palavras-chave (a) e (c) tiveram o índice de 5,74\% e 5,47\%, respectivamente.

O gráfico da Figura 01 mostra o desenvolvimento das pesquisas relacionadas a cada palavra-chave ao longo dos anos. Nota-se que a temática (c) sempre teve grande destaque nas pesquisas. Porém nos seis últimos anos, os estudos relacionados a (a) teve um vultoso crescimento, chegando a se igualar aos estudos relacionados à (c), devido à implantação de uma política de controle da pressão para evitar perdas reais nos sistemas de saneamento por parte das concessionárias (DARVINI et al. 2015). 


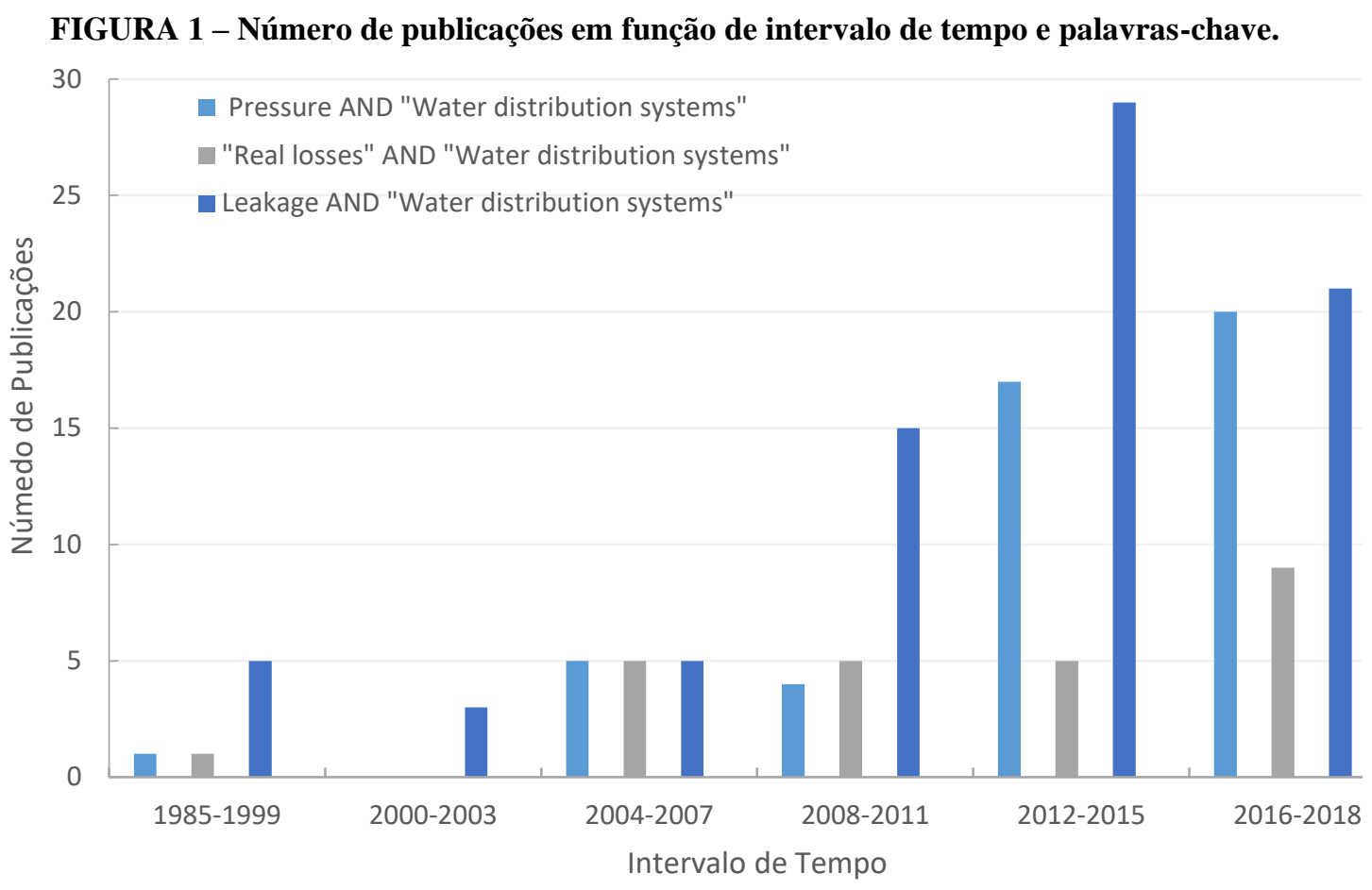

Fonte: Autoria Própria.

O número de artigos na temática selecionados por país é apresentado na Figura 2. Países da Ásia tem expressivo número de artigos, com destaque para a China e também com participações de Israel e Arábia Saudita. Na América, grande número de artigos publicados são oriundos dos Estados Unidos, mas com participação significativa do Canadá e do Brasil.

Em termos gerais, os artigos oriundos da Europa são 62,67\% de todo o levantamento. Isso se justifica considerando que esse continente é constituído de países históricos, e que tem a maioria das redes antigas de distribuição de água (BOANO et al. 2015). Através do levantamento, constata-se que foram de países desse continente os primeiros e principais estudos realizados em função das demandas de manutenções nas suas redes. 
FIGURA 2 - Número de publicações em função dos países com publicações nas palavras-chave em estudo.

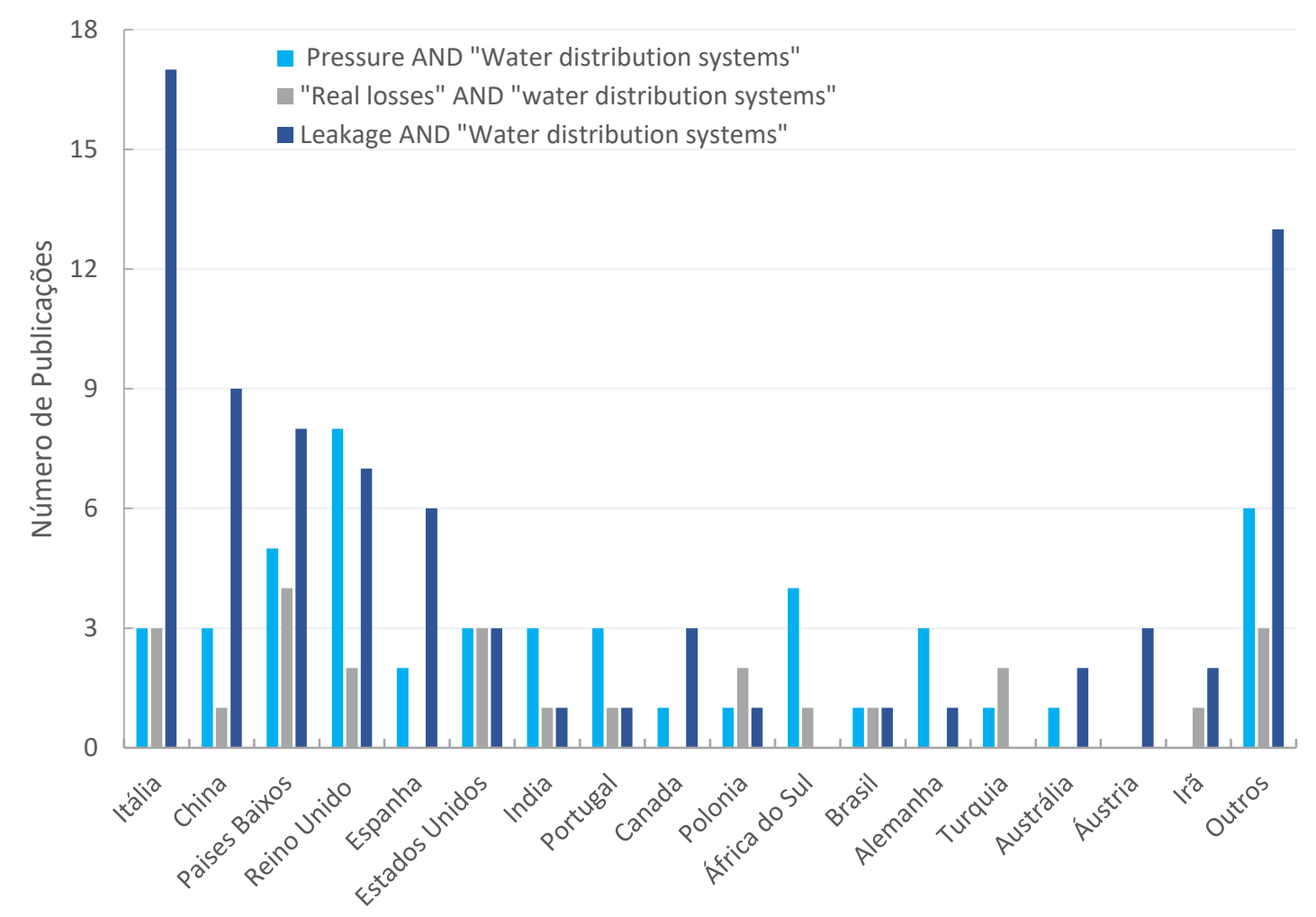

Fonte: Autoria Própria.

A avaliação dos periódicos internacionais com maior frequência de publicação é discriminada na Tabela 03. A revista Journal of Water Resources Planning and Management foi a de maior ocorrência de publicações com o percentual de 23,33\%, seguido da revista Procedia Engineering com 20\%. Quando são considerados somente os últimos cinco anos, a revista Procedia Engineering tem o número de 33,33\% de publicações de artigos, o que assegura que nos últimos cinco anos ela foi a revista que mais publicou artigos relativos ao tópico em estudo. 
TABELA 2 - Principais ocorrências de periódicos internacionais com publicação nas palavraschave selecionadas

\begin{tabular}{l|c|c|c|c}
\hline \multicolumn{1}{c}{ Principais Periódicos } & $\begin{array}{c}\text { Pressure AND } \\
\text { "Water } \\
\text { distribution } \\
\text { systems" (a) }\end{array}$ & $\begin{array}{c}\text { "Real losses" } \\
\text { AND "water } \\
\text { distribution } \\
\text { systems" (b) }\end{array}$ & $\begin{array}{c}\text { Leakage AND } \\
\text { "Water } \\
\text { distribution } \\
\text { systems" (c) }\end{array}$ & Total \\
\hline $\begin{array}{l}\text { Journal of Water Resources Planning } \\
\text { and Management }\end{array}$ & 15 & 4 & 16 & $\mathbf{3 5}$ \\
\hline Procedia Engineering & 13 & 2 & 15 & $\mathbf{3 0}$ \\
\hline Water Resources Management & 7 & 5 & 12 & $\mathbf{2 4}$ \\
\hline $\begin{array}{l}\text { Water Science and Technology: } \\
\text { Water Supply }\end{array}$ & 0 & 1 & 7 & $\mathbf{8}$ \\
\hline Journal of Hydraulic Engineering & 1 & 0 & 5 & $\mathbf{6}$ \\
\hline $\begin{array}{l}\text { Journal - American Water Works } \\
\text { Association }\end{array}$ & 1 & 1 & 2 & $\mathbf{4}$ \\
\hline Outras & 9 & 10 & 18 & $\mathbf{4 3}$ \\
\hline Total & $\mathbf{4 6}$ & $\mathbf{2 5}$ & $\mathbf{7 9}$ & $\mathbf{1 5 0}$ \\
\hline
\end{tabular}

Fonte: Autoria Própria.

As principais vertentes identificadas após a realização da leitura minuciosa dos resumos dos artigos selecionados ao fim do processo descrito na metodologia são apresentadas na Tabela 4. As Figuras 3, 4 e 5 mostram em termos quantitativos a ocorrência de cada vertente em função das palavras-chave.

Os temas que mais tem sido objeto de pesquisa dos autores foram a Otimização da Localização e Número de VRP, com 25 ocorrências, Avaliação de Vazamentos em Sistemas de Distribuição de Água e Gerenciamento de Pressões, com 24 estudos verificados cada, e Otimização por Algoritmos ou Sistemas Computacionais, com 21 artigos. Por outro lado, as vertentes que foram menos exploradas são: Modelos de Calibração para Otimização de Redes, com 8 ocorrências, Reabilitação da Rede, com 6 estudos verificados, Relação do Material da Tubulação na ocorrência de vazamentos, com 2 artigos e Foco na Economia de Água e Sustentabilidade com apenas 1 artigo. 
TABELA 3 - Relação das Vertentes identificadas em função das palavras-chave após a leitura dos resumos dos artigos

\begin{tabular}{|c|c|}
\hline Palavras-Chave & Vertente Identificada \\
\hline $\begin{array}{l}\text { Pressure AND "Water } \\
\text { distribution systems" }\end{array}$ & $\begin{array}{l}\text { - Relação Pressão-Vazamento } \\
\text { - Otimização da Localização e Número de Válvulas Redutoras de } \\
\text { Pressão (VRP) } \\
\text { - Gerenciamento de Pressões } \\
\text { - Otimização por algoritmos ou sistemas computacionais }\end{array}$ \\
\hline $\begin{array}{l}\text { "Real losses" AND } \\
\text { "Water distribution } \\
\text { systems" }\end{array}$ & $\begin{array}{l}\text { - Controle de Perdas Reais } \\
\text { - Foco na Economia de Água e Sustentabilidade } \\
\text { - Detecção de perdas reais } \\
\text { - Otimização por algoritmos ou sistemas computacionais }\end{array}$ \\
\hline $\begin{array}{l}\text { Leakage AND "Water } \\
\text { distribution systems" }\end{array}$ & $\begin{array}{l}\text { - Relação do material da tubulação na ocorrência de vazamentos } \\
\text { • Reabilitação da Rede } \\
\text { - Avaliação de Vazamentos } \\
\text { • Modelos de Calibração para otimização de redes } \\
\text { - Otimização por algoritmos ou sistemas computacionais }\end{array}$ \\
\hline
\end{tabular}

Fonte: Autoria Própria.

FIGURA 3 - Número de publicações em função das vertentes identificadas nas palavras-chave: Pressure AND "Water distribution systems".

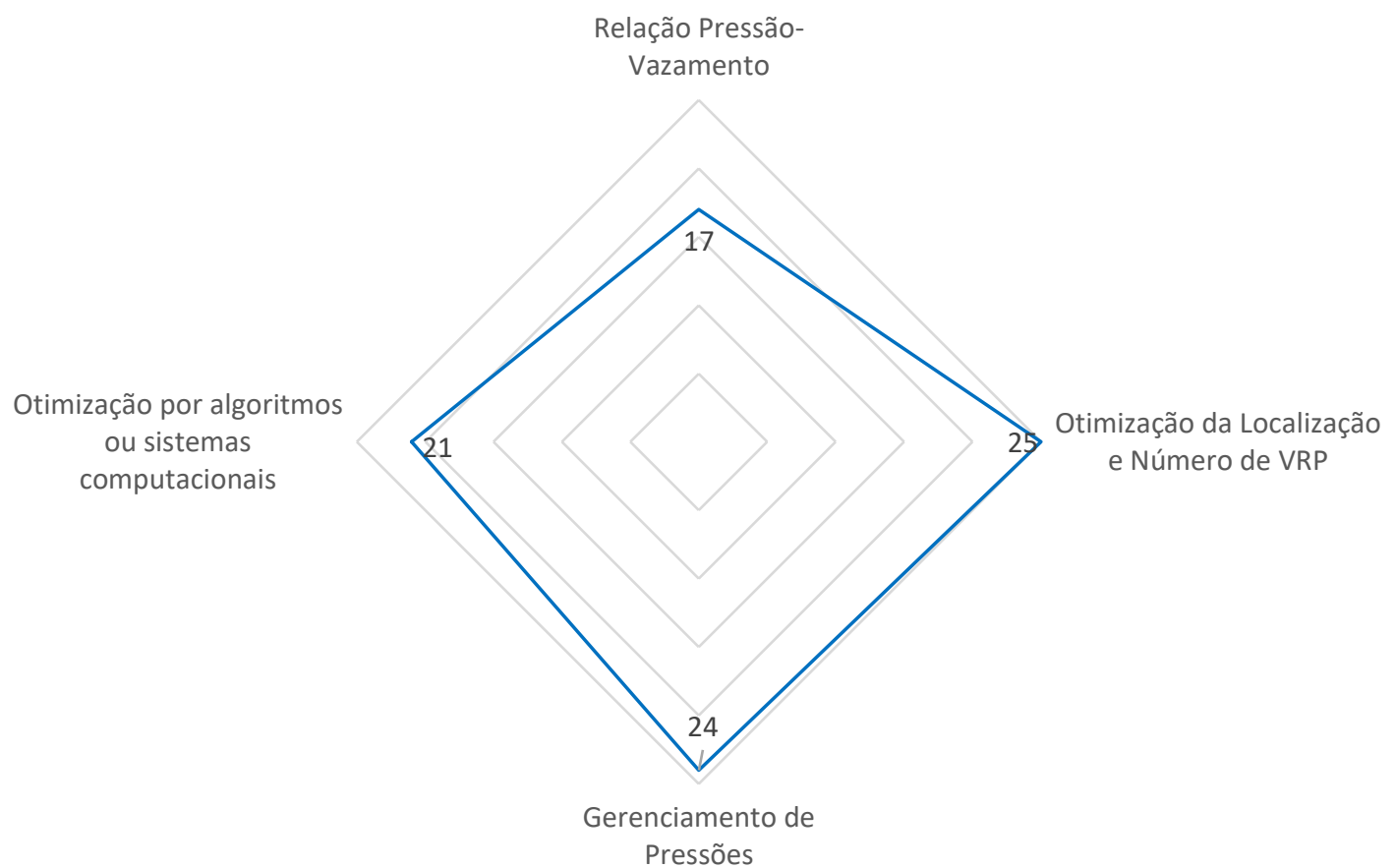

Fonte: Autoria Própria. 
FIGURA 4 - Número de publicações em função das vertentes identificadas nas palavras-chave: "Real losses" AND "Water distribution systems".

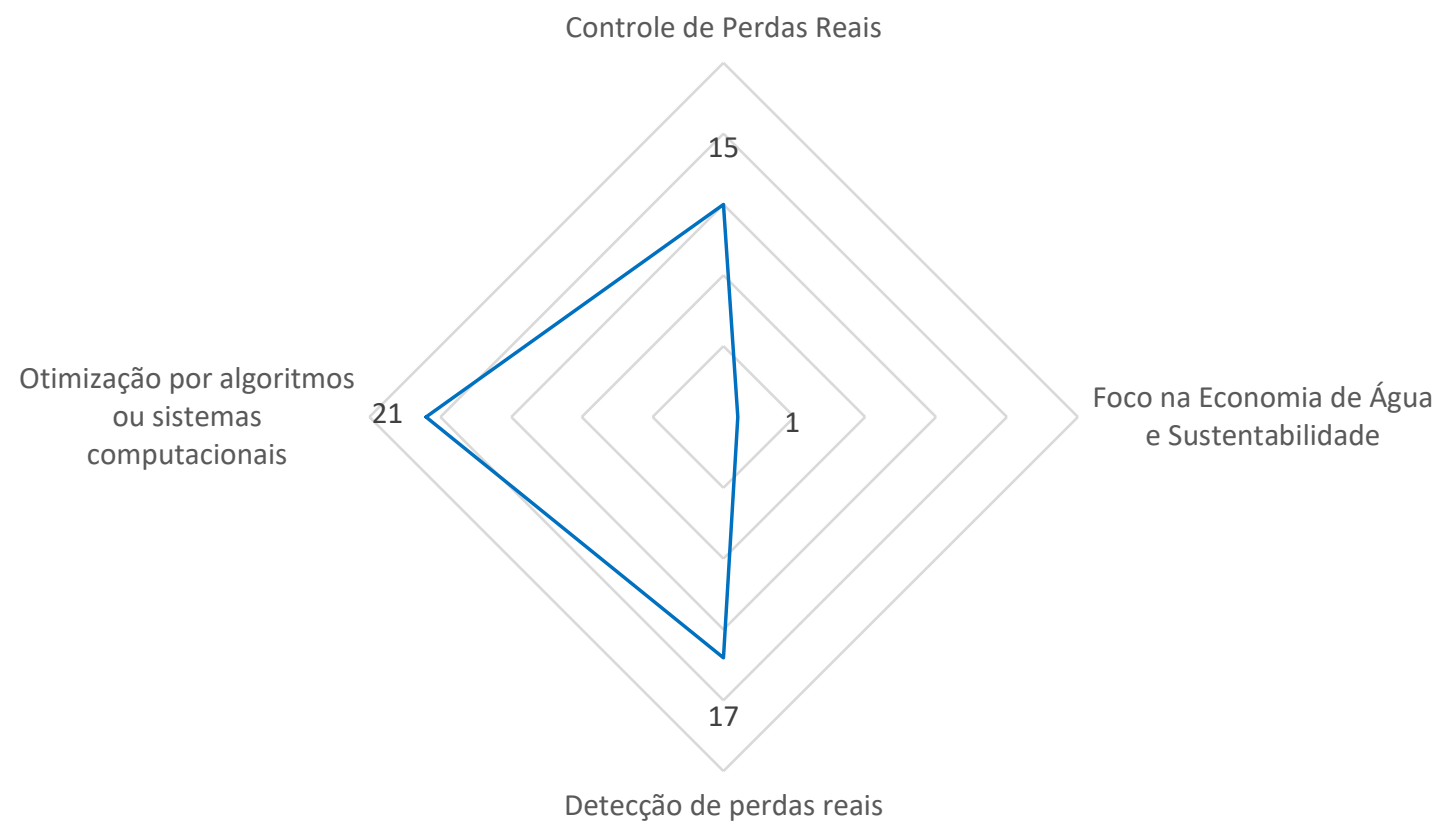

Fonte: Autoria Própria.

FIGURA 5 - Número de publicações em função das vertentes identificadas nas palavras-chave: Leakage AND "Water distribution systems".

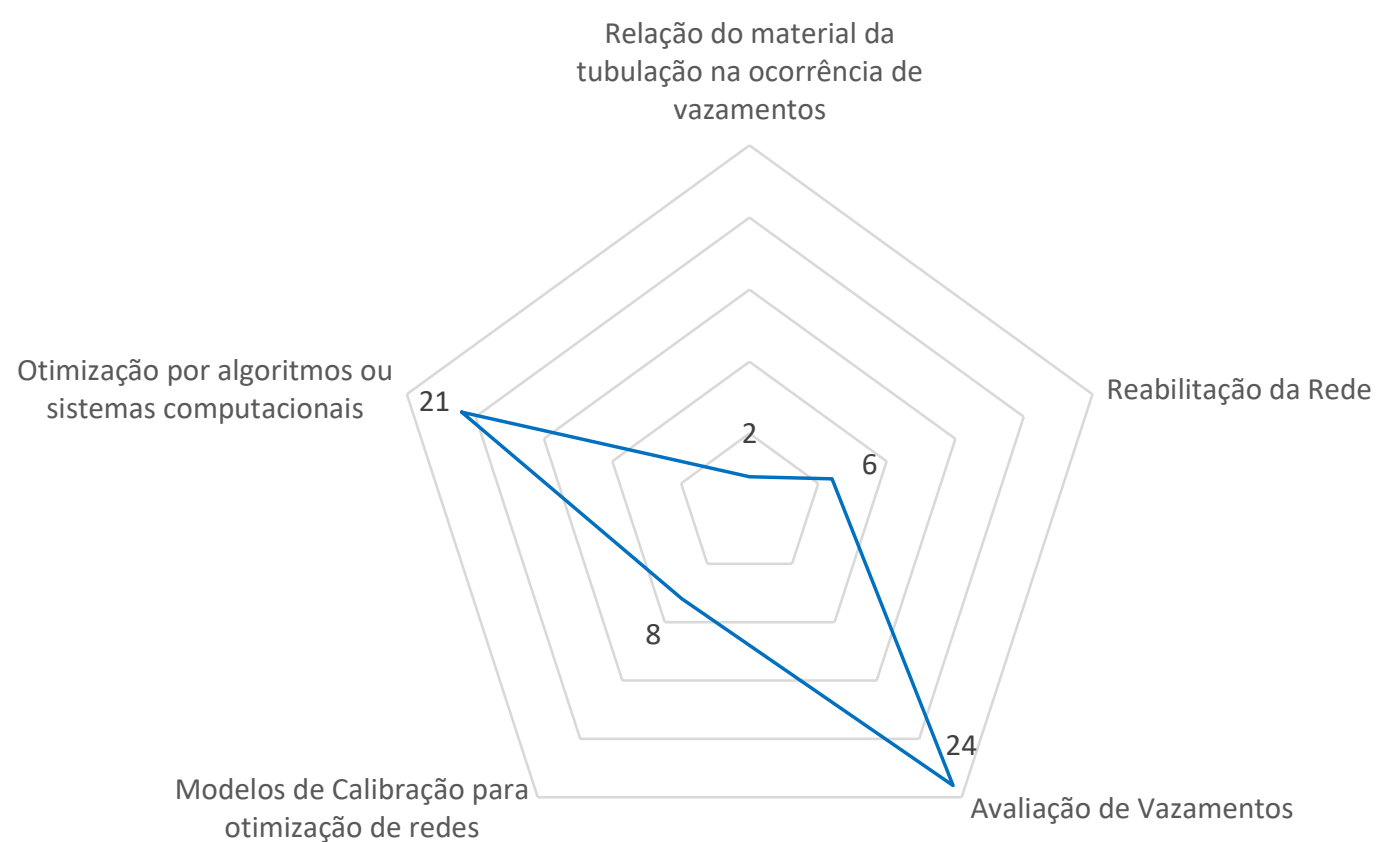

Fonte: Autoria Própria. 
Devido à simplicidade da aplicação do uso de VRP nas redes para o controle das pressões, geralmente a aplicação desse método é feito de forma empírica, sem uma avaliação aprofundada (SAMIR et.al (2017). Essa foi uma lacuna observada por vários autores que gerou grande interesse em estudos envolvendo técnicas de otimização nos sistemas para avaliação desse .

A utilização de VRP no controle dos vazamentos segue como o principal meio para controle das perdas nas redes. A novidade dos últimos estudos é a utilização de simuladores virtuais juntamente com algoritmos computacionais para a otimização da redução desses vazamentos e na determinação de sua localização.

\section{CONCLUSÕES}

Através do uso do Mapping Study na realização da revisão bibliográfica, foi possível alcançar resultados suficientes e amplos dentro do tema proposto. Dentro da metodologia aplicada, identificou-se o histórico de pesquisas e os periódicos relativos ao tema.

As pesquisas que relacionam o uso de VRP junto ao controle e avaliação dos vazamentos e o gerenciamento das pressões são as mais volumosas dentro do levantamento. No campo interdisciplinar, as pesquisas com mais destaques envolvem a otimização de redes por algoritmos e sistemas computacionais. Estudos relativos à relação do material das tubulações com a ocorrência de vazamentos, o Foco na Economia de Água e Sustentabilidade e os Modelos de Calibração para otimização de redes são as vertentes menos exploradas. Conforme já avaliado, essas pesquisas que envolvem controle de perdas reais ganharam importância devido eventos atípicos de grandes secas que geraram escassez de água nas grandes metrópoles nos últimos anos.

\section{REFERÊNCIAS}

ABES - Associação Brasileira de Engenharia Sanitária e Ambiental - Perdas em sistemas de abastecimento de água: diagnóstico, potencial de ganhos com sua redução e propostas de medidas para o efetivo combate, São Paulo, 2013.

BAILEY, J.; BUDGEN, D.; TURNER, M; KITCHENHAM, B; BRERETON, P.; LINKMON, S. Evidence relating to Object Oriented software design: A survey. First International Symposium on Empirical Software Engineering and Measurement. Computer Society, 2007.

BOANO, F; SCIBETTA, M; RIDOLFI, L; GIUSTOLISI, O. Water distribution system modeling and optimization: a case study. Procedia Engineering. V.119. p. 719-724. 2015.

BONFIM, W. B.; BALDIN, V.; PEREIRA, R. R.; PAULA, H. M. Lama residual de usinas de concreto: características e aplicações na confecção de blocos. REEC - Revista Eletrônica de Engenharia Civil, v. 13, n. 2, p. 32 - 43, 2017.

DARVINI, G; SOLDINI, L. Pressure control for WDS management. A case study. Procedia Engineering. V.119. p. 984-993. 2015.

FERNANDES, F. G; FERNANDES, G. R; PAULA, H. M. Mapeamento Sistemático de Referências da Análise de placas compostas por materiais heterogênos através de uma 
abordagem em multiescala. REEC - Revista Eletrônica de Engenharia Civil, v. 14, n. 2, p. 176 $188,2018$.

NICOLINI, M; ZOVATTO, L. Optimal Location and Control of Pressure Reducing Valves in Water Networks. Journal of Water Resources Planning and Management. V.135. p. 178-187. 2009.

PAULA, H. M. D.; ILHA, M. S. D. O. Uso da Moringa oleifera no tratamento de águas residuárias de usinas de concreto: mapeamento sistemático. REEC - Revista Eletrônica de Engenharia Civil, v. 11, n. 1, p. 50 - 60, 2016.

PECCI, F; ABRAHAM, E; STOIANOV, I. Mathematical programming methods for pressure management in water distribution systems. Procedia Engineering. V.119. p. 937-946. 2015..

RODRIGUES, K. C.; MESQUITA, H. C.; EDUARDO, R. C.; PAULA, H. M. Mapeamento sistemático de referências do uso do BIM na compatibilização de projetos na construção civil. REEC - Revista Eletrônica de Engenharia Civil, v. 13, n. 1, p. 219 - 239, 2017.

SAMIR, N; KANSOH, R.; ELBARKI, W; FLEIFLE, A. Pressure control for minimizing leakage in water distribution systems. Alexandria Engineering Journal, v. 56, p. 601 - 612, 2017.

SISTEMA NACIONAL DE INFORMAÇÕES SOBRE SANEAMENTO (SNIS).

Diagnóstico dos serviços de água e esgotos - 2016. Brasília, 2016.

WHO - World Health Organization, Leakage Management and Control - A Best Practice Manual, WHO, Geneva, 2001. 